\title{
Dark Current Reduction in Stacked-Type CMOS-APS for Charged Particle Imaging
}

\author{
Isao Takayanagi, Member, IEEE, Junichi Nakamura, Eric R. Fossum, Fellow, IEEE, Kazuhide Nagashima, \\ Takuya Kunihoro, and Hisayoshi Yurimoto
}

\begin{abstract}
A stacked CMOS-active pixel sensor (APS) with a newly devised pixel structure for charged particle detection has been developed. At low operation temperatures $(<200 \mathrm{~K})$, the dark current of the CMOS-APS is determined by the hot carrier effect. A twin well CMOS pixel with a p-MOS readout and n-MOS reset circuit achieves low leakage current as low as $5 \times 10^{-8} \mathrm{~V} / \mathrm{s}$ at the pixel electrode under liquid nitrogen temperature of $77 \mathrm{~K}$. The total read noise floor of $0.1 \mathrm{mV}_{\text {rms }}$ at the pixel electrode was obtained by nondestructive readout correlated double sampling (CDS) with the CDS interval of $21 \mathrm{~s}$.
\end{abstract}

Index Terms-Active pixel sensor (APS), CMOS image sensor, correlated double sampling (CDS), detectors, fixed-pattern noise (FPN), leakage current, mass spectroscopy.

\section{INTRODUCTION}

C HARGED particle detectors have been used for mass spectrometry systems and a two-dimensional charged particle imaging system has been employed to reconstruct a spectrograph or spatial distribution of the ion density. A photo-plate or a fluorescent screen with a micro-channel plate (MCP) is commonly used as the two-dimensional detector for mass spectrographs and ion micrographs [1]. Due to very small thermal noise in the MCP with high multiplication gain, this imaging system provides a high sensitivity as a single ion can be detected. However, total performance is determined by the characteristics of the screen. Because of the nonlinear response of the screen, it is difficult to obtain good linearity and quantitative accuracy when many ions are simultaneously incident on a pixel. The quantum efficiency of MCP is determined by the aperture ratio of the micro-channel and it yields signal loss in ion-to-electron conversion. Gain degradation in MCP over time is another problem in actual usage.

Matsumoto et al. [2] reported that spatial resolution, linearity, and dynamic range of ion micrographs and mass spectrographs were greatly improved by using a Stacked AMI (Amplified MOS Imager) compared with the conventional system. They employed a stacked imager approach in which a surface electrode located at the top of the pixel detects incoming ions directly and integrates the signal charge. The signal charge is readout by scanning pixels by on-chip readout electronics.

Manuscript received April 18, 2002; revised October 24, 2002. The review of this paper was arranged by Editor A. Theuwissen.

I. Takayanagi and J. Nakamura are with the Japan Imaging Design Center, Micron Japan, Tokyo 105-6234, Japan (e-mail: istakayanagi@micron.com).

E. R. Fossum is with Micron Technology, Inc., Pasadena, CA 91101-1758 USA (e-mail: eric_fossum@hotmail.com).

K. Nagashima, T. Kunihoro, and H. Yurimoto are with the Tokyo Institute of

Technology, Tokyo 152-8551, Japan (e-mail: yuri@geo.titech.a.jp).

Digital Object Identifier 10.1109/TED.2002.806963
Since no nonlinear devices, such as a florescent screen, are contained in this signal chain, linearity in ion conversion can be improved. In addition to the superior performance characteristics, a use of the stacked CMOS-APS provides several advantages, including low voltage operation, low power consumption, long lifetime, stable detectability against mass number and robustness against environment [2], [3]. However, the sensitivity of the stacked imagers was much lower than that of the MCP approach, so further improvement in noise reduction is required.

In Section II, basic imager architecture and operation of the stacked imager for charged particle detection are introduced. A problem with dark current of the conventional three-transistor NMOS active pixel under low operation temperatures is also explained in this section with experimental results. In Section III, dark current of the conventional three-transistor NMOS active pixel is analyzed with experimental results obtained from test pixel structures to make the problems clear. Then a stacked CMOS-APS with a newly devised pixel structure is presented in Section IV. Fabrication and characterization results of the new pixel are presented in Section V. The new pixel achieves much lower leakage current under low operation temperature and higher sensitivity than those of conventional stacked pixels, which permits longer integration time with low read noise, thereby yielding wider dynamic range.

\section{Charged Particle IMAging FOR MASS SPECTROMETER}

\section{A. Stacked Pixel Approach}

A schematic configuration of a conventional n-MOS stacked pixel for charged particle detection is shown in Fig. 1. The pixel consists of a top metal electrode and three transistors, which include a readout transistor $\mathrm{M}_{\mathrm{RD}}$, a row select transistor $\mathrm{M}_{\mathrm{SEL}}$, and a reset transistor $\mathrm{M}_{\mathrm{RS}}$. The top electrode is exposed by eliminating the surface passivation layer, so that the signal ions are irradiated directly.

The top electrode is connected to the gate electrode of $\mathrm{M}_{\mathrm{RD}}$ and a source electrode of $\mathrm{M}_{\mathrm{RS}}$. At the beginning of charge detection period, the top electrode is reset to the reset voltage $\mathrm{V}_{\mathrm{RS}}$ by turning $\mathrm{M}_{\mathrm{RS}}$ on. Then, signal ions are irradiated onto the top electrode. Charged particles with acceleration energy of several $\mathrm{keV}$ generate multiple secondary electrons when they hit the surface electrode. An anode plate is located about $1 \mathrm{~mm}$ apart above the periphery of the imaging array and thus provides an aperture for the imaging area. By applying voltage to the anode plate of about several tens of volts, the secondary electrons generated by collision of incident ions are collected by the anode electrode. Since the kinetic energy of several keV 


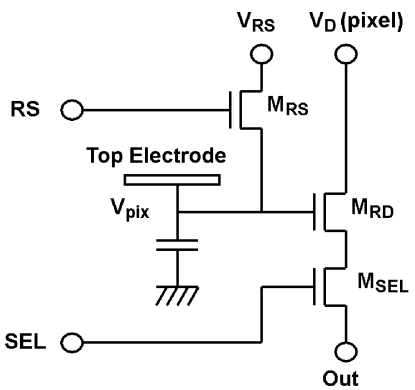

Fig. 1. Circuit configuration of a conventional stacked-type charged particle detection pixel. Voltage of the top electrode $\mathrm{V}_{\text {pix }}$ is read out through a source-follower circuit formed by $\mathrm{M}_{\mathrm{RD}}$ and an external load circuit which is connected to the Out node.

of the incident ions is much larger than the electrical potential of the anode electrode, distortion caused by the anode electrode is negligible. Charge of the implanted ions and the positive charge corresponding to the emitted secondary electrons are accumulated on a storage capacitor, thereby increasing the potential of the top electrode. A multiplication factor of larger than unity is thus expected. The amount of the accumulated charge can be estimated by reading out the increase of the top electrode potential through a source-follower circuit with its load being not shown in the figure.

The feature of this stacked pixel structure is that the detecting part of charged particles and the signal readout part are separated. It results in the protection of the device from the high energy collision of the charged particles, so that long lifetime with stable characteristics can be obtained. A high fill factor is another advantage of the stacked type pixel, which gives high quantum efficiency. It improves the signal-to-noise ratio and quantitative accuracy.

The charged particle imager has been used for a secondary ion mass spectrometer (SIMS) system. A block diagram of our ion imaging system is shown in Fig. 2. The imager is installed inside SIMS and held in vacuum, so that the ion image is directly focused on the top electrodes of the imager. The signal from the imager is amplified by a low noise operational amplifier and converted to digital data, which is fed to a $\mathrm{PC}$ to reconstruct an image. The imager is installed in a vacuum chamber and cooled by liquid $\mathrm{N}_{2}$ to achieve long integration time by reducing thermally generated dark current.

In order to decrease readout noise, reset noise suppression using nondestructive readout correlated double sampling (NDRO-CDS) is used [4]. The operation of NDRO-CDS is shown in Fig. 3. First, the imager is reset by turning the reset transistor $\mathrm{M}_{\mathrm{RS}}$ on, and an offset signal that includes fixed pattern noise (FPN) and reset noise is readout nondestructively and transferred to a frame memory. After an integration period, the signal is readout again. Since the reset noise components in both images are identical, it can be suppressed by subtracting the offset image. In addition, since the second signal is also readout nondestructively, integration can be continued if the amount of signal is not large enough; namely the sensor is capable of monitoring the integration condition.

Compared to the CCD, this nondestructive readout capability of CMOS active pixel is one of advantages in applications of stacked-type imager. In stacked type CCDs, it is difficult to

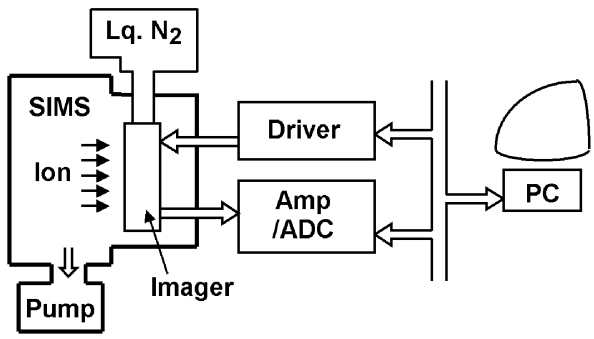

Fig. 2. Schematic block diagram of the ion imaging system. The stacked-type imager is installed in a projection-type SIMS (secondary ion mass spectrometer).

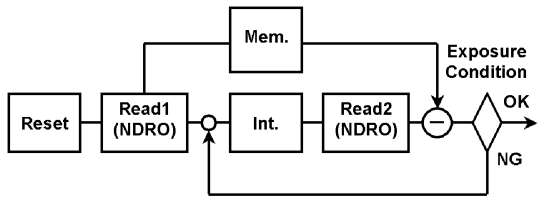

Fig. 3. Operation sequence of the nondestructive readout correlated double sampling (NDRO-CDS). Signal processing is performed by an external computer illustrated in Fig. 2.

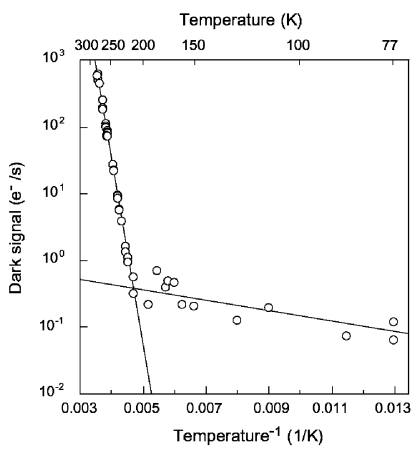

Fig. 4. Temperature dependence of dark current of a conventional charged particle imager.

suppress reset noise, since the stacked electrode and a charge storage part of a pixel are connected by a metal contact, which yields incomplete charge transfer between the charge storage part and a CCD register and results in the reset noise in every transfer operation [5].

Another significant noise source is the dark current. Because of low signal intensity in actual usage of ion imaging, the integration time often exceeds one hour to obtain quantitative accuracy. Therefore, a cooling system to reduce dark current must be implemented. Fig. 4 shows a measurement result of temperature dependence of the dark current in a charged particle imager with a conventional pixel structure that consists of n-MOS transistors located in a common p-well [6]. The dark current depends on thermal generation model with activation energy of about $0.55 \mathrm{eV}$ from $200 \mathrm{~K}$ to room temperature. The activation energy is in good agreement with half of the bandgap of silicon. However below $200 \mathrm{~K}$, the temperature dependence becomes weak with an equivalent activation energy of $0.015 \mathrm{eV}$ and a dark current of $0.1 \mathrm{e}^{-} / \mathrm{s}$ is measured even at $77 \mathrm{~K}$, which suggests different mechanism in dark current generation under low operation temperatures. Assuming an integration time of one hour, the dark current of $0.1 e^{-/ \mathrm{s} \text { results in several }}$ 


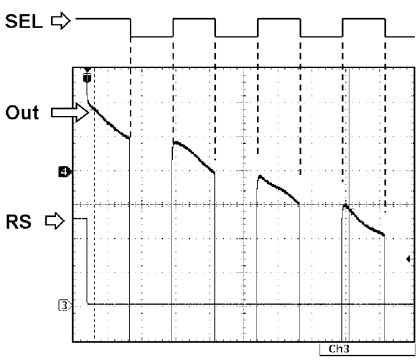

Fig. 5. Oscilloscope waveform of dark current integration, which was obtained from a test pixel structure at $100 \mathrm{~K}$. The schematic configuration of the pixel is the conventional three-transistor circuit shown in Fig. 1.

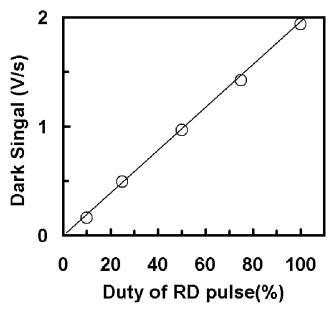

Fig. 6. Relationship between dark current under operation temperature of $100 \mathrm{~K}$ and the duty of the select pulse SEL. Dark current is expressed as the voltage drift in $\mathrm{V}_{\mathrm{pix}} / \mathrm{s}$.

hundreds of dark electrons, which causes significant noise due to its quantum fluctuation and variations in pixels.

\section{DARK CURRENT ANALYSIS}

In order to study generation mechanism of the dark current under low operation temperatures, test pixel structures were designed and fabricated in $0.8 \mu \mathrm{m}$ CMOS process. Fig. 5 shows a measurement result of dark current generation in a test pixel structure operated at $100 \mathrm{~K}$. The pixel configuration is the conventional n-MOS circuit as shown in Fig. 1. After the pixel was reset by a reset pulse RS, dark current was integrated with applying periodical select pulses SEL. As shown in Fig. 5, the dark current is mostly generated during the readout period when the select pulse is set at high.

In Fig. 6, dark current values are plotted versus duty of the select pulse. The vertical axis of the figure denotes the dark current and is expressed as a voltage drift/s at the pixel electrode, Vpix. As shown in the figure, the dark current is proportional to the duty of the select pulse, and the value is large compared to that of the general-purpose imagers. In general-purpose imagers, dark current is determined by thermal generation and the value is commonly in the order of a few $\sim$ several tens of $\mathrm{mV} / \mathrm{s}$. From these facts, it means that there exists a certain different mechanism in dark current generation under low temperatures and a leakage due to a hot carrier effect is the most possible cause.

It is known that minority carrier injection due to the hot carrier effect contributes to the dark current [7]. In Fig. 7, drain voltage dependences of the dark current obtained from test pixel structures with different gate length of $\mathrm{M}_{\mathrm{RD}}$ are shown. The duty of the select pulse was set at $2.5 \times 10^{-5}$, which equivalently corresponds to an operation condition of a $\sim 40 \mathrm{k}$ pixel imager. The dark current depends on the drain voltage exponentially,

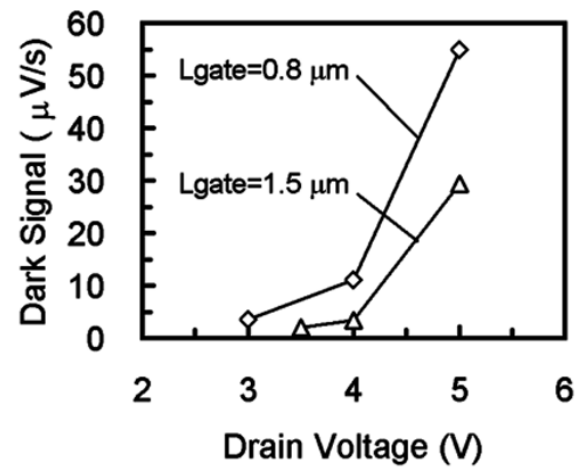

Fig. 7. Drain voltage dependence of the dark current of pixels with gate length of $0.8 \mu \mathrm{m}$ and $1.5 \mu \mathrm{m}$ at $100 \mathrm{~K}$. The duty of the select pulse was set at $2.5 \times$ $10^{-5}$.

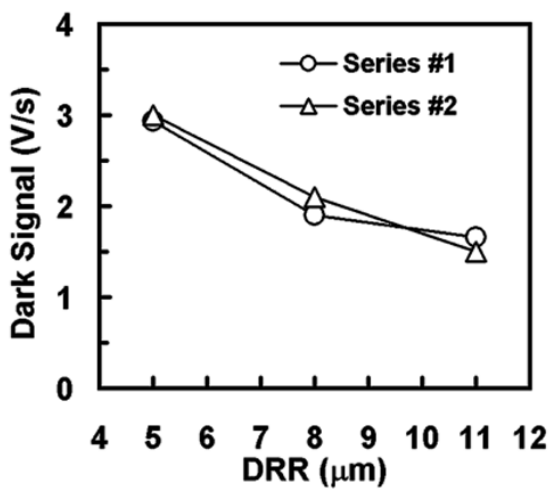

Fig. 8. Relationship between measured dark current at $100 \mathrm{~K}$ and the distance between $M_{R D}$ and the $n+$ source of $M_{R S}$.

and decreases with increased gate length of the $\mathrm{M}_{\mathrm{RD}}$. These experimental results clearly suggest the contribution from the hot carrier effect. Because of low temperature, mobility of channel electrons increases, which should enhance the hot carrier effect.

The hot carriers in the readout transistor can emit photons [8] and the optically generated carriers result in excess dark current [9], [10], [12]. If the photogenerated carrier contributes dark current generation, it should depend on the distance between the readout transistor $\mathrm{M}_{\mathrm{RD}}$ and the $\mathrm{n}^{+}$-floating electrode of $\mathrm{M}_{\mathrm{RS}}$. In order to identify contribution from the photo generated carriers, three pixel structures with the distance of $5 \mu \mathrm{m}, 8 \mu \mathrm{m}$, and $11 \mu \mathrm{m}$ were fabricated and tested. In Fig. 8, dark current versus the distance between $\mathrm{M}_{\mathrm{RD}}$ and $\mathrm{n}^{+}$-diffusion, DRR, is plotted and it is seen that the dark current decreases to about a half with the decrease in DRR from $5 \mu \mathrm{m}$ to $11 \mu \mathrm{m}$. The emitted photons also generate minority carries in the p-well region outside of the depletion layer around the $\mathrm{n}^{+}$-floating diffusion and a part of the generated minority carriers can diffuse into the floating diffusion. Therefore the experiment was not sufficient to estimate quantitative amount of photon emission, but it identifies contribution from the electrons generated by emitted photons.

From these facts, it can be concluded that it is difficult to achieve complete suppression of the dark current by this approach due to the pixel size limitation, although increasing the gate length of the readout transistor and/or increasing the distance between readout transistor and the $\mathrm{n}^{+}$-sense node reduces dark current. 


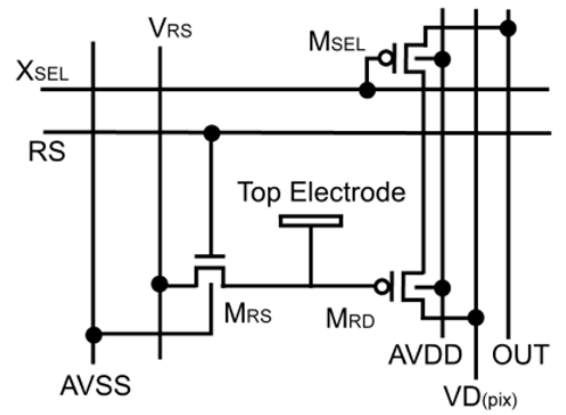

Fig. 9. Circuit configuration of the proposed CMOS pixel. The pixel consists of a pMOS readout circuit and an nMOS reset circuit. Transistor size of $M_{R D}$ is $1.8 \mu \mathrm{m} / 1.1 \mu \mathrm{m}(\mathrm{W} / \mathrm{L})$.

\section{PiXel DESIGN FOR DARK CURRENT REDUCTION}

Assuming the leakage models mentioned above, we propose a new pixel structure. The points of the design of this pixel are suppression of the hot carrier effect and avoidance of carrier diffusion into the floating diffusion. Circuit configuration of the pixel is shown in Fig. 9. The pixel consists of p-MOS readout and n-MOS reset circuits. The hot carrier effect in p-MOS transistors is lower than that of n-MOS transistors due to lower carrier mobility [11]. In addition to smaller hot carrier generation at the readout transistor, the dark current component from photo generation can also be suppressed by implementing twin-wells into this pixel circuit. A schematic cross-sectional view of the pixel is illustrated in Fig. 10. The photo-generated carriers are mostly collected by the well regions and drained through well contacts, while only electrons generated very close to the $\mathrm{n}^{+}$-source region of $\mathrm{M}_{\mathrm{RS}}$ have a possibility to diffuse into the region. Therefore, the low hot-carrier effect and low dark current are expected with this pixel.

Since the accumulated signal has positive charge in this particular ion detection application, the pixel voltage increases with accumulated signal, and thus the top electrode should be initialized at a low voltage to extend dynamic range. Therefore, an n-MOS reset transistor and a p-MOS readout transistor are preferable.

\section{FABRICATION AND CHARACTERIZATION RESULTS}

In order to confirm dark current reduction capability of the proposed pixel, a new charged particle imager was fabricated and tested. Fig. 11 shows the developed charged particle detection imager. The imager was fabricated in $0.8 \mu \mathrm{m}$ twin-well CMOS technology. Pixel size is $20 \mu \mathrm{m} \times 20 \mu \mathrm{m}$ and the number of effective pixels is $600 \times 576$, yielding an image area of $12.00 \mathrm{~mm} \times 11.52 \mathrm{~mm}$. Because of the stacked structure, a high fill factor of $88 \%$ has been achieved, and highly sensitive ion detection is possible. A reset inhibit function is implemented to achieve NDRO-CDS operation. Read noise of the imager at $100 \mathrm{~K}$ is measured to be $0.1 \mathrm{mV}_{\mathrm{rms}}$ referred to the top electrode voltage with NDRO-CDS where the CDS interval of $21 \mathrm{~s}$ and $20 \mathrm{kHz}$ pixel rate are used. With a design value of pixel capacitance of $14 \mathrm{fF}$, the equivalent noise electron number is estimated to be $9 e^{-}$. The measured read noise voltage includes

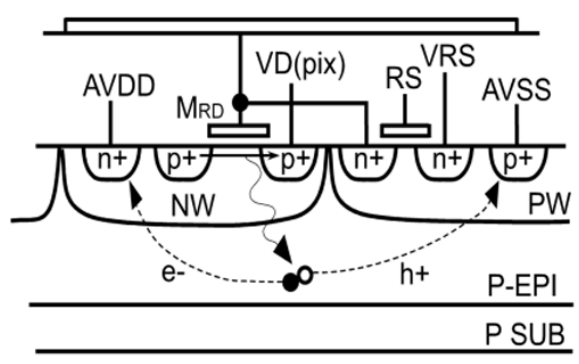

Fig. 10. Schematic cross-sectional view of the new pixel. The select transistor $\mathrm{M}_{\mathrm{SEL}}$ is not shown in this figure. A black circle and a white circle denote an electron and a hole generated by a photon emitted at $\mathrm{M}_{\mathrm{RD}}$.

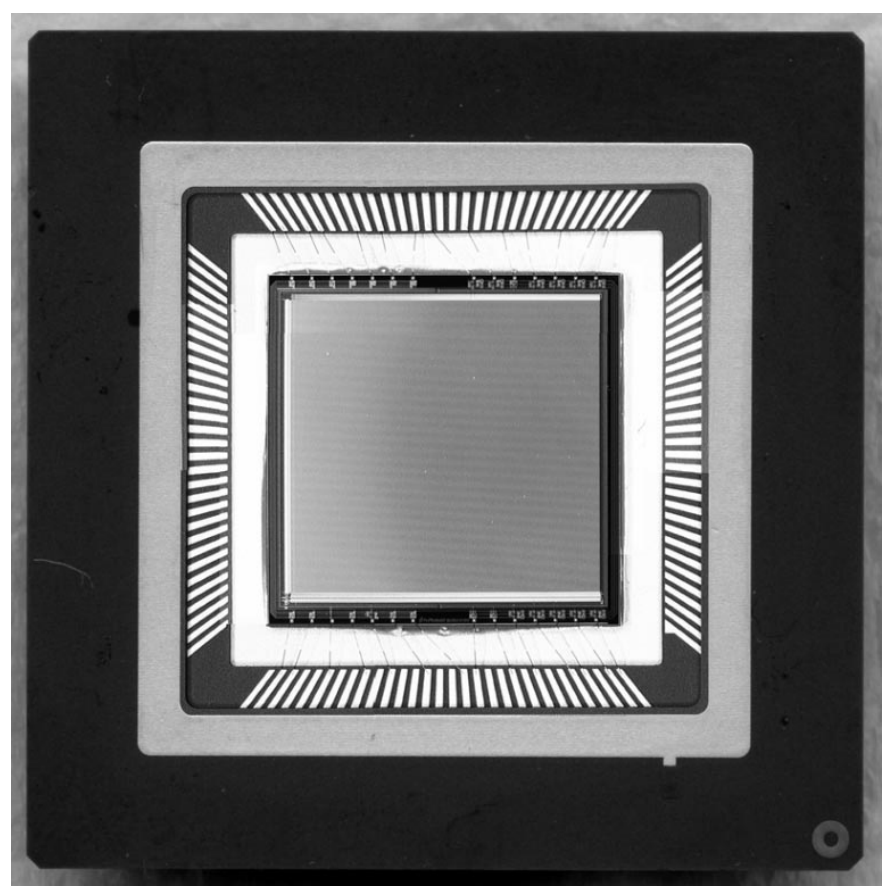

Fig. 11. Chip photo of the newly developed charged particle imager assembled in a PGA package.

those from dark current FPN and external circuits. In conventional CDS operation, reset-noise voltage is estimated from an equation of $\sqrt{2 k T / C}$, where $\mathrm{k}$ and T denote Boltzmann's constant and absolute temperature, respectively, and is calculated to be $0.44 \mathrm{~m} V_{r m s}$. Therefore, the experimental result shows that noise floor was reduced to be less than $1 / 4$ of the conventional reset noise.

The relationship between the dark signal rate at $77 \mathrm{~K}$ and the drain voltage of $\mathrm{M}_{\mathrm{RD}}$ is shown in Fig. 12. The dark current signal has been significantly reduced to less than $1 \times 10^{-7} \mathrm{~V} / \mathrm{s}$ at the pixel electrode with the proposed pixel. The dark current is approximately two orders smaller than that of the conventional n-MOS pixel. Since the dynamic range and linearity of the ion conversion characteristics were not degraded when the drain voltage is larger than $3.5 \mathrm{~V}$, the drain voltage of $3.5 \mathrm{~V}$ is considered as a lower limit. Dark signal as low as $5 \times 10^{-8}$ $\mathrm{V} / \mathrm{s}$ permits very long signal integration period. For example, when the integration time is $1 \mathrm{~h}$, dark charge is estimated to be $16 \mathrm{e}^{-}$per pixel with a design value of pixel capacitance of $14 \mathrm{fF}$. 


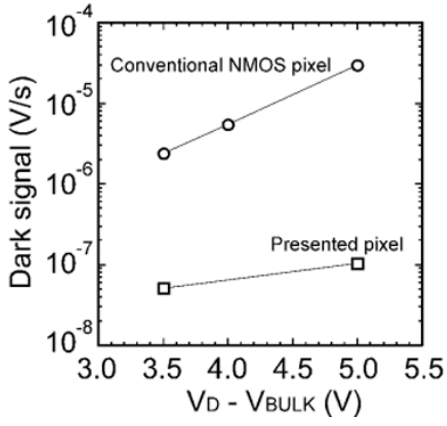

Fig. 12. Measured dark currents of the new pixel. Measured dark currents obtained from a pixel test structure of the conventional nMOS pixel are also plotted. Duty of the select pulse was $2.4 \times 10^{-6}$ and the pixels were operated at $77 \mathrm{~K}$.

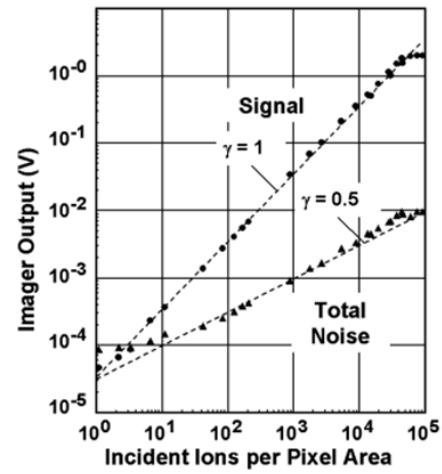

Fig. 13. Ion conversion characteristics of the imager at $77 \mathrm{~K}$. Incident ions are $\mathrm{Al}^{+}$ions with acceleration energy of $10 \mathrm{keV}$. The horizontal axis represents the number of total incident ions per pixel area measured by a Faraday Cup. The amount of signal ions was controlled by changing the integration time. The vertical axis denotes output voltage of the imager. Values of signal and noise voltages are the averaged value and the root mean square values in a $100 \times 100$ pixel area, respectively.

Imager output at $77 \mathrm{~K}$ versus $\mathrm{Al}^{+}$incident ion with $10 \mathrm{keV}$ energy is shown in Fig. 13. The horizontal axis denotes the number of incident ion per pixel area of $400 \mu \mathrm{m}^{2}$ and averaged signals 'Signal' and root mean square values of total noise 'Total Noise' are plotted. The NDRO-CDS operation was used in the measurement. Total noise includes dark-current fixed-pattern noise, sensitivity variations of pixels and system noise generated in the following signal chain. The input ion numbers were obtained from Faraday Cup measurement. The averaged signal output increases in proportion to the incident ion number and are very close to $\gamma=1$ line drawn by a dotted line, which shows good linearity of the imager in a region below an output saturation level of $2.0 \mathrm{~V}$ corresponding to $5 \times 10^{4}$ ions. Noise floor of the system was measured to be $85 \mu \mathrm{V}_{\text {rms }}$ at imager output that equivalently corresponds to about two input ions. The total noise increases in proportion to the square root of the number of incident ions in the range above ten input ions and below $10^{4}$ ions, thus having a slope of $\gamma=0.5$, which indicates that the noise is shot noise of the incident ions. The total noise becomes larger than that of the shot noise model when incident ions are larger than $10^{4}$ due to sensitivity variations of pixels. Sensitivity nonuniformity of this imager is approximately $0.5 \%$ $\mathrm{rms}$. The cross point between signal line and the shot noise line
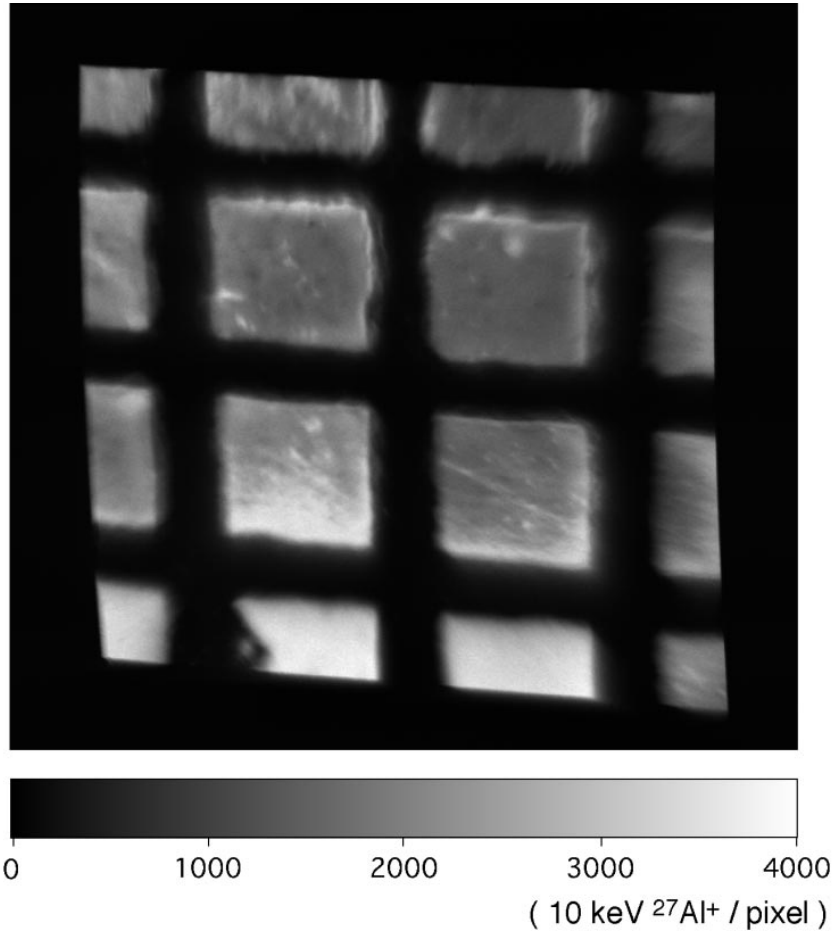

Fig. 14. Obtained $\mathrm{Al}^{+}$ion image from a SIMS using the imager.

TABLE I

SPECIFICATIONS OF THE CHARgED PARTICLE IMAGER

\begin{tabular}{ll}
\hline Pixel size & \multicolumn{1}{l}{$20 \mu \mathrm{m} \times 20 \mu \mathrm{m}$} \\
Number of pixels & $600 \times 576$ \\
Fill factor & $88 \%$ \\
Digital power supply & $5 \mathrm{~V}$ \\
Analog power supply & $3.5 \mathrm{~V}, 1.5 \mathrm{~V}$ \\
Noise floor & $<3$ ions $\left(10 \mathrm{keV} \mathrm{Al}^{+}\right.$ion $)$ \\
$\quad$ (with NDRO-CDS) & \\
Saturation & 50,000 ions $\left(10 \mathrm{keV} \mathrm{Al}{ }^{+}\right.$ion $)$ \\
Dark current & $<16 \mathrm{e}^{\circ} / \mathrm{hour}(100 \mathrm{~K})$ \\
Chip size & $14.0 \mathrm{~mm} \times 13.9 \mathrm{~mm}$ \\
\hline
\end{tabular}

is very close to one incident ion, which means high quantum efficiency of the pixel due to the high fill factor. It is also found that the secondary electrons does not affect ion shot noise. Since the multiplication factor due to emission of secondary electrons is estimated to be about 4.5 through the course of this work, actual integrated charge is 4.5 times larger than that of the incident ions.

A reproduced image of $\mathrm{Al}^{+}$ion density distribution obtained with the imager is shown in Fig. 14. The targeted sample is an aluminum plate that is covered with $\mathrm{Cu}$ grids with its pitch of $25 \mu \mathrm{m}$ and $5 \mu \mathrm{m}$ width. The reproduced image shows good resolution characteristics of the imager with high sharpness.

Specifications of the imager are summarized in Table I. 


\section{CONCLUSION}

A low dark current stacked CMOS APS for charged particle detection has been developed using a newly devised pixel. Dark current of CMOS APS under low operation temperatures is determined by hot carrier effect. A use of a p-MOS FET for a readout transistor reduces a hot carrier effect inside the pixel and the twin-well pixel structure avoids photo generated carrier diffusion into the floating sense node. As a result, the dark current in the low-temperature region was extremely decreased. The pixel configuration also reduces read noise because flicker noise of a p-MOSFET is smaller than that of an n-MOSFET. The low dark current, low read-out noise and high fill factor of the imager permit accurate charged particle image capturing for applications such as ion and electron imaging.

The ion imaging system utilizing the stacked CMOS APS achieves wide dynamic range of $87 \mathrm{~dB}$ with high linearity.

In future developments, further sensitivity improvement to reach the sensitivity of the MCP based imaging system is possible by increasing the conversion factor in readout electronics and the multiplication factor at the top electrode.

\section{REFERENCES}

[1] A. Benninghoven, Secondary Ion Mass Spectrometry: Basic Concepts, Instrumental Aspects, Applications and Trends. New York: Wiley, 1987.

[2] K. Matsumoto, H. Yurimoto, K. Kosaka, T. Nakamura, and S. Sueno, "A novel imager for secondary ion mass spectrometry," IEEE Trans. Electron Devices, vol. 40, pp. 82-85, Jan. 1993.

[3] S. Fuerstenau, G. Soli, T. Cunningham, B. Hancock, B. Pain, and M. Sinha, "Active pixel sensors for charge detection mass spectrometry," in Proc. 46th ASMS Conf. Mass Spectroscopy and Allied Topics, Orlando, FL, May 1998.

[4] I. Takayanagi, J. Nakamura, H. Yurimoto, T. Kunihiro, K. Nagashima, and K. Kosaka, "A Stacked CMOS APS for charge particle detection and its noise performance," in Proc. Progr. IEEE Workshop on ChargeCoupled Devices and Advanced Image Sensors, R18, June 1999.

[5] Y. Matsunaga, F. Hatori, H. Tango, and O. Yoshida, "Analysis of signal to noise ratio of photoconductive layered solid-stage imaging devices," IEEE Trans. Electron Devices, vol. 42, pp. 38-42, Jan. 1995.

[6] T. Kunihiro, K. Nagashima, I. Takayanagi, J. Nakamura, K. Kosaka, and H. Yurimoto, "Noise characteristics of stacked CMOS active pixel sensor for charged particles," Nucl. Instrum. Meth. Phys. Res., vol. A 470, pp. 512-519, 2001.

[7] J. Matsunaga and S. Kohyama, "Evidence for impact-ionized electron in substrate of n-channel MOS structure," Appl. Phys. Lett., vol. 33, no. 4, pp. 335-337, 1978.

[8] P. A. Childs, R. A. Stuart, and W. Eccleston, "Evidence of optical generation of minority carriers from saturated MOS transistors," Solid-State Electron., vol. 26, no. 7, pp. 685-688, 1983.

[9] S. Tam, F. C. Hsu, C. Hu, and S. Muller, "Spatially resolved observation of visible-light emission from Si MOSFET'," IEEE Electron Device Lett., vol. EDL-4, pp. 386-388, Oct. 1983.

[10] S. Tam and C. Hu, "Hot-electron-induced photon and photocarrie generation in silicon MOSFET's," IEEE Trans. Electron Devices, vol. ED-31, pp. 1264-1273, Sept. 1984.

[11] T. Ohzone, H. Iwata, Y. Uraoka, and S. Odanaka, "Photon-energy distribution of hot-carrier photoemission from LOCOS and trenchisolated MOSFET's," Solid-State Electron., vol. 37, no. 7, pp. 1421-1428, 1994.

[12] C. C. Wang and C. G. Sodini, "The effect of hot carriers on the operation of CMOS active pixel sensors," IEDM Tech. Dig., pp. 563-566, 2001.

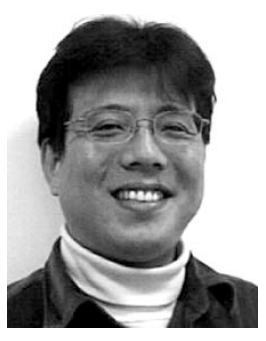

Isao Takayanagi (M'02) was born in Tokyo, Japan, on June 6,1962. He received the M.S. degree in physics from Gakushuin University, Tokyo, in 1987.

He joined the Olympus Optical Co., Ltd., in 1987. In 2000, he joined the Photobit Corporation (now Micron Imaging, an image sensor development division of Micron Technology, Inc.). Since 2001, he has been with Japan Imaging Design Center of Micron Japan, Ltd. He has been engaged in research and development on solid-state image sensors.

Mr. Takayanagi is a member of the Japan Society of Applied Physics and the Institute of Image Information and Television Engineers of Japan.

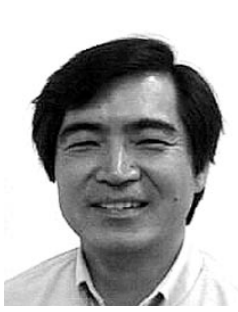

Junichi Nakamura received the B.S.E.E and M.S.E.E. degrees in electronics engineering from Tokyo Institute of Technology, Tokyo, Japan, in 1979 and 1981, respectively, and the Ph.D. degree in electronics engineering from the University of Tokyo in 2000

He joined Olympus Optical Co., Tokyo, in 1981. After working on optical image processing, he was involved in the development of active pixel sensors, including static induction transistor (SIT) image sensors and MOS type image sensors. From September 1993 to October 1996, he was resident at the Jet Propulsion Laboratory (JPL), California Institute of Technology, Pasadena, as Distinguished Visiting Scientist. At JPL, he worked on on-chip signal processing circuits including image smoothing circuit using a neuron MOSFET and a current-mode analog-to-digital converter, with the Advanced Imager and Focal-plane Technology Group led by Dr. E. R. Fossum. He joined Photobit Corporation, Pasadena, CA, in 2000, where he led several custom sensor developments. Since November 2001, he has been with Japan Imaging Design Center, Micron Japan, Ltd.

Dr. Nakamura is a member of the Institute of Image Information and Television Engineers of Japan. He served as Technical Program Chairman for the 1995 and 1999 IEEE Workshop on Charge-coupled Devices and Advanced Image Sensors.

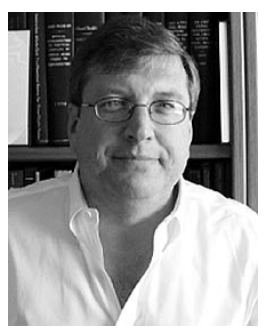

Eric. R. Fossum (S'80-M'84-SM'91-F'98) was born and raised in Connecticut. He received the B.S. degree in physics and engineering from Trinity College, Hartford, CT, in 1979 and the Ph.D. degree in electrical engineering from Yale University, New Haven, CT, in 1984.

As a member of Columbia University's Electrical Engineering faculty from 1984-1990, he and his students performed research on CCD focal-plane image processing and high speed III-V CCDs. In 1990, Dr. Fossum joined the NASA Jet Propulsion Laboratory, California Institute of Technology, Pasadena, and managed JPL's image sensor and focal-plane technology research and advanced development. He led the invention and development of CMOS active pixel sensor (APS) camera-on-a-chip and subsequent transfer of the technology to US industry. In 1996, he joined Photobit Corporation as Chief Scientist, a company he helped found in 1995, and became CEO of Photobit Technology Corporation in 2000. While at Photobit, he and his staff commercialized the camera-on-a-chip which has been used in Logitech $^{\circledR}$ and Intel ${ }^{\circledR}$ PC cameras, ultra-low-power sensors enabling the swallowable "pill-camera," and very-high-speed, high-resolution sensors used for Hollywood special effects such as in The Mummy Returns and Pearl Harbor. In late 2001, Photobit was acquired by Micron Technology, Inc. and he is presently a Senior Micron Fellow. He has also served as Adjunct Professor of electrical engineering at the University of California, Los Angeles (UCLA), and is currently Adjunct Professor of electrical engineering-electrophysics at the University of Southern California (USC), Los Angeles, and teaches undergraduate physical electronics. He has served as primary adviser to $12 \mathrm{Ph} . \mathrm{D}$. candidates. He has published 230 technical papers and holds 53 U.S. patents. 
Dr. Fossum received Yale's Becton Prize in 1984, the IBM Faculty Development Award in 1984, the National Science Foundation Presidential Young Investigator Award in 1986, the JPL Lew Allen Award for Excellence in 1992, and the NASA Exceptional Achievement Medal in 1996. He was inducted into the U.S. Space Foundation Technology Hall of Fame in 1999. He founded the biannual IEEE Workshops on CCDs and Advanced Image Sensors and the SPIE Conferences on Infrared Readout Electronics. He has served on program committees for the IEDM, ISSCC, and SPIE conferences. He has served as associate editor for IEEE TRANSACTIONS ON VLSI, guest editor for IEEE JOURNAL OF SOLID STATE CIRCUITS, and was Guest Editor-in-Chief for IEEE TRANSACTIONS ON ELECTRON DEVICES Special Issue on Solid-State Image Sensors published in October 1997. He was recently named to the Board of Fellows for Trinity College, CT where he also serves on the college's Engineering Advisory Committee.

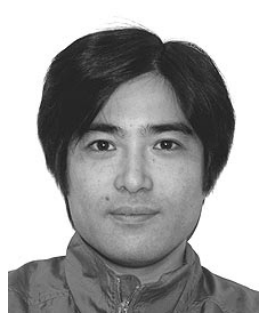

Kazuhide Nagashima was born in Tokyo, Japan, on April 9, 1972. He received the B.S., M.S., and Ph.D degrees in earth and planetary sciences from Tokyo Institute of Technology in 1996, 1998, and 2001, respectively.

Since 2001 he has been a Researcher with Venture Business Laboratory, Tokyo Institute of Technology, where he works on isotope study using SIMS about meteorites.

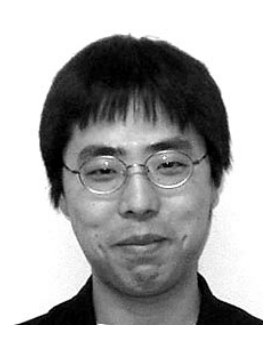

Takuya Kunihiro was born in Yokohama, Japan, on August 7, 1973. He received the Ph.D. degree in earth and planetary sciences from Tokyo Institute of Technology, Tokyo, Japan, in 2001.

Since 2002, he has worked as a Postdoctoral Fellow with the University of California, Los Angeles. He has conducted research on secondary ion mass spectrometry and the origin of the solar system.

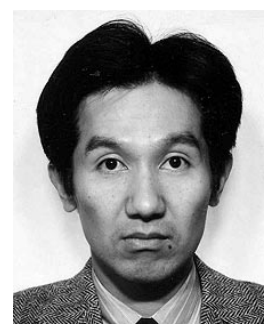

Hisayoshi Yurimoto was born in Wakayama, Japan, on March 12, 1958. He received the D.Sc. degree in geoscience from the University of Tsukuba, Japan, in 1985.

From 1985 to 1994, he was a Postdoctoral Fellow and an Assistant Professor with the University of Tsukuba. He joined the Department of Earth and Planetary Sciences, Tokyo Institute of Technology as an Associate Professor in 1994. He is mainly interested in the origin of the earth and planets and secondary ion mass spectrometry. 